\title{
van Stockum-Bonnor Spacetimes of Rigidly Rotating Dust
}

\author{
Łukasz Bratek, ${ }^{1}$ Joanna Jałocha, ${ }^{1}$ and Marek Kutschera ${ }^{1,2}$ \\ ${ }^{1}$ Institute of Nuclear Physics PAN, Radzikowskego 152, 31-342 Kraków, Poland. \\ ${ }^{2}$ Jagellonian University, Institute of Physics, Reymonta 4, 30-059 Kraków, Poland.
}

(Dated: 27 Feb 2007)

\begin{abstract}
Stationary, axisymmetric and asymptotically flat spacetimes of dust of which trajectories are integral curves of the time translation Killing vector are investigated. The flow has no Newtonian limit. Asymptotic flatness implies the existence of singularities of the curvature scalar that are distributions and that are not isolated from regularity regions of the flow. The singularities are closely related to the presence of additional stresses that contribute negative active mass to the total (Komar) mass, which is zero for asymptotically flat spacetimes. Several families of solutions are constructed.
\end{abstract}

\section{INTRODUCTION}

We study stationary, axisymmetric and asymptotically flat spacetimes of dust in free fall along integral curves of the time translation Killing vector. For reasons which will become clear later, we shall call this motion 'van Stockum flow'. Despite the flow is a rigid rotation with zero angular velocity with respect to asymptotically static observers, the squared vorticity scalar (which for the particular flow equals, up to a constant factor, the proper energy density of dust) in contrast to Newtonian physics, does not vanish. Angular velocity of the flow with respect to locally non-rotating observers numerically equals the angular velocity of dragging of inertial frames, while angular velocity of matter in linearized gravity is many orders of magnitude greater. This shows that van Stockum flow is ultra-relativistic even in the limit of negligible density.

We demonstrate that asymptotic flatness implies the existence of curvature singularities that have distributional character, spatial measure zero, and are not isolated from regularity regions. The singularities are closely related to additional weird stresses with negative active mass. Total mass of such spacetimes is necessarily zero, which has already been conjectured by Bonnor [3], and total angular momentum is determined by the amplitude of the dipole in a multipole expansion.

We construct two classes of asymptotically flat solutions and a class of asymptotically non-flat solutions. Bonnor's solutions [2], [3] belong to the first two, and the van Stockum solution [1] to the third.

\section{VAN STOCKUM FLOW}

We shall focus on axisymmetric, stationary and asymptotically flat spacetimes of dust flowing along opened integral curves of the time translation Killing vector $\boldsymbol{\xi}$. By asymptotic flatness the axial symmetry Killing vector $\boldsymbol{\eta}$, of which integral lines are closed, vanishes on the symmetry axis at least for radii sufficiently large. Consequently, i) $\eta_{[\alpha} \xi_{\beta} \xi_{\mu ; \nu]}=0$ and $\xi_{[\alpha} \eta_{\beta} \eta_{\mu ; \nu]}=0$ at least at one point. The energy-momentum tensor is proportional to $\xi_{\mu} \xi_{\nu}$, hence ii) $\xi^{\mu} R_{\mu}{ }^{[\nu} \xi^{\alpha} \eta^{\beta]}=0$ and $\eta^{\mu} R_{\mu}{ }^{[\nu} \xi^{\alpha} \eta^{\beta]}=0$ on the basis of Einstein's equations. In addition, we assume iii) $[\boldsymbol{\xi}, \boldsymbol{\eta}]=0$. Under these three assumptions a theorem proved in [4] guarantees that, maybe apart from isolated points $(\rho, z)$, there exist a coordinate system in which the line element takes the general form

$$
\mathrm{d} s^{2}=-V(\mathrm{~d} t-K \mathrm{~d} \phi)^{2}+V^{-1} \rho^{2} \mathrm{~d} \phi^{2}+e^{2 \Psi}\left(\mathrm{d} \rho^{2}+\Lambda \mathrm{d} z^{2}\right),
$$

where $V, K, \Psi$ and $\Lambda$ are structure functions of two variables $\rho$ and $z$. In these coordinates $\boldsymbol{\xi}$ and $\boldsymbol{\eta}$ attain the particularly simple form $\xi^{\mu}=\delta_{t}^{\mu}$ and $\eta^{\mu}=\delta_{\phi}^{\mu}$.

The four-velocity of van Stockum flow reads $\boldsymbol{u}=Z \boldsymbol{\xi}$, thus $Z^{-2}=-\xi^{\mu} \xi_{\mu}$ and $\xi^{\mu} Z_{, \mu}=0$. Killing equations imply $u_{(\mu ; \nu)}=\xi_{(\mu} Z_{, \nu)}$, hence the expansion scalar $u_{; \mu}^{\mu}=0$. On projecting $u_{(\mu ; \nu)}$ onto the $\boldsymbol{u}$-orthogonal subspace and taking the traceless part, one infers the shear tensor vanishes identically, as well. van Stockum flow is therefore rigid. Despite the fact and that angular velocity of the flow also vanishes $(\boldsymbol{u} \propto \boldsymbol{\xi})$, the vorticity scalar $\omega^{2}$ does not, where

$$
\omega^{2}=\frac{1}{4} \frac{(\boldsymbol{\xi} \boldsymbol{\xi})^{2}(\nabla S)^{2}}{(\boldsymbol{\xi} \boldsymbol{\eta})^{2}-\boldsymbol{\xi}^{2} \eta^{2}}, \quad S=\frac{\boldsymbol{\xi} \boldsymbol{\eta}}{\boldsymbol{\xi} \boldsymbol{\xi}}
$$

Typeset by REVTEX 


\section{A. Equations of van Stockum flow}

By definition of the flow, the energy-momentum tensor reads $T_{\mu \nu}=\mathcal{D} Z^{2} \xi_{\mu} \xi_{\nu}$, where $\mathcal{D}$ is the proper energy density such that $\xi^{\mu} \mathcal{D}_{, \mu}=0=\eta^{\mu} \mathcal{D}_{, \mu}$. Einstein's equations and the contracted Bianchi identity imply the flow is continuous and geodesic. As $u_{; \mu}^{\mu}=0$, the continuity equation $\left(\mathcal{D} Z \xi^{\mu}\right)_{; \mu}=0$ is satisfied identically. In addition, $u^{\nu} u_{\mu ; \nu}=-u^{\nu} Z \xi_{\nu ; \mu}$. The geodesic equation $u^{\nu} u_{\mu ; \nu}=0$ will be satisfied if $0=-u^{\nu} u_{\nu ; \mu}+u^{\nu} u_{\nu}(\ln Z)_{, \mu}$. As $u^{\mu} u_{\mu} \equiv-1$, $Z$ must be constant. This, in turn, implies $V$ is also constant and, without loss of generality, we may set $V \equiv 1$. On defining $K^{\mu \nu}=\boldsymbol{\xi}^{2} \eta^{\mu} \eta^{\nu}+2 \boldsymbol{\xi} \boldsymbol{\eta} \xi^{\mu} \eta^{\nu}+\boldsymbol{\eta}^{2} \xi^{\mu} \xi^{\nu}$ we obtain for dust $K^{\mu \nu}\left(T_{\mu \nu}-T g_{\mu \nu} / 2\right)=0$, then Einstein's equations imply $K^{\mu \nu} R_{\mu \nu}=0$ or $\rho e^{-2 \Psi} \partial_{\rho} \ln \sqrt{|\Lambda|}=0$ in coordinates, hence $\Lambda=\Lambda(z)$. If so, the form of (2.1) allows us to set $\Lambda(z) \equiv 1$. We have thus shown that the line element of van Stockum flow reads

$$
\mathrm{d} s^{2}=-\mathrm{d} t^{2}+2 K(\rho, z) \mathrm{d} t \mathrm{~d} \phi+\left(\rho^{2}-K^{2}(\rho, z)\right) \mathrm{d} \phi^{2}+e^{2 \Psi(\rho, z)}\left(\mathrm{d} \rho^{2}+\mathrm{d} z^{2}\right) .
$$

Let $E^{\mu}{ }_{\nu}=R^{\mu}{ }_{\nu}-\frac{1}{2} R \delta^{\mu}{ }_{\nu}-8 \pi T^{\mu}{ }_{\nu}$, then $E^{\rho}{ }_{\rho}=0$ and $E^{\rho}{ }_{z}=0$ yield the following relations

$$
\Psi_{, \rho}=\frac{K_{, z}^{2}-K_{, \rho}^{2}}{4 \rho}, \quad \Psi_{, z}=-\frac{K_{, \rho} K_{, z}}{2 \rho}
$$

The integrability condition $\Psi_{, \rho z}=\Psi_{, z \rho}$ imposes on $K$ the elliptic constraint

$$
\mathcal{L} K=0, \quad \mathcal{L}=\partial_{\rho}^{2}-\frac{1}{\rho} \partial_{\rho}+\partial_{z}^{2} .
$$

Provided (2.3) and (2.4) are satisfied, the other components of $E^{\mu}{ }_{\nu}$, but $E_{t}{ }_{t}$ and $E^{t}{ }_{\phi}$, vanish identically. The latter two will also vanish for $\mathcal{C}^{2}$ solutions (we stress, the reservation 'for $\mathcal{C}^{2}$ solutions' is necessary) if only

$$
\mathcal{D}=e^{-2 \Psi} \frac{K_{, \rho}^{2}+K_{, z}^{2}}{8 \pi \rho^{2}}
$$

\section{ASYMPTOTIC FLATNESS AND CURVATURE SINGULARITIES}

A function $K^{\epsilon}$ is a regularized profile of a solution $K$ of (2.4) in an open subset $\mathcal{V}$ of the plane $(\rho, z)$ if $K^{\epsilon} \in \mathcal{C}^{\infty}(\mathcal{V})$ and $K^{\epsilon} \rightarrow K$ as $\epsilon \rightarrow 0$ almost everywhere in $\mathcal{V}$. We shall denote by $\mathcal{I}$ the set of all irregularity points of van Stockum flow, that is, a subset of $\mathbb{R}^{3}$ where $\mathcal{L} K$ does not exist for solutions in the sense that $\lim _{\epsilon \rightarrow 0} \int_{\mathcal{I}_{\delta}} \mathrm{d} m f \mathcal{L} K^{\epsilon} \neq 0$ for any $\delta>0$, where $\mathcal{I} \subset \mathcal{I}_{\delta}, 0<\operatorname{dist}\left(\partial \mathcal{I}_{\delta}, \mathcal{I}\right)<\delta, e^{2 \Psi} f=\rho^{-1} K_{, \rho}^{\epsilon}$ or $\rho^{-2} K^{\epsilon}$ and $\mathrm{d} m=e^{2 \Psi} \rho \mathrm{d} \rho \mathrm{d} \phi \mathrm{d} z$. In particular, $K \notin \mathcal{C}^{2}(\mathcal{I})$. We recall the result of the theory of elliptic equations that $\mathcal{I} / \mathcal{S}^{1}$ has measure zero in the plane $(\rho, z)$.

In what follows we shall prove that $\mathcal{I} \neq \emptyset$ for asymptotically flat van-Stockum spacetimes. For if we suppose for contradiction that $\mathcal{I}=\emptyset$, then inside a ball $\mathcal{B}_{\mathrm{R}} \subset \mathbb{R}^{3}$ bounded by a two-sphere $\mathcal{S}_{\mathrm{R}}$ of radius $R$ and concentric with the origin

$$
\int_{\mathcal{B}_{\mathrm{R}}} \mathcal{D} e^{2 \Psi} \rho \mathrm{d} \rho \wedge \mathrm{d} \phi \wedge \mathrm{d} z \stackrel{\mathcal{L} K=0}{=} \frac{1}{8 \pi} \int_{\mathcal{S}_{\mathrm{R}}} \frac{K}{\rho}\left(K_{, z} \mathrm{~d} \rho-K_{, \rho} \mathrm{d} z\right) \wedge \mathrm{d} \phi \equiv \frac{1}{8 \pi} \int_{\mathcal{S}_{\mathrm{R}}} \frac{K \partial_{r} K}{\sin \theta} \mathrm{d} \phi \wedge \mathrm{d} \theta
$$

in virtue of the Stokes theorem, provided $\left(K^{2}\right)_{, r}=o(\sin \theta),(r \sin \theta=\rho, r \cos \theta=z)$. By asymptotic flatness $K \sim 2 J r^{-1} \sin ^{2} \theta$ as $r \rightarrow \infty$, hence, for $\mathrm{R}$ sufficiently large, the surface integral on the right-hand side is negative and tends to 0 as $\mathrm{R} \rightarrow \infty$, while the volume integral is positive, a contradiction, thus indeed $\mathcal{I} \neq \emptyset$.

The surface integral (3.1) coincides in the limit $\mathrm{R} \rightarrow \infty$ with the total (Komar) mass $M$, which is zero. Indeed, for asymptotically flat spacetimes with metric (2.2), $M$ is given by ${ }^{1}$

$$
M=-\frac{1}{8 \pi} \lim _{\mathrm{R} \rightarrow \infty} \int_{\mathcal{S}_{\mathrm{R}}} \frac{1}{2} \sqrt{-g} \epsilon_{\alpha \beta \mu \nu} \nabla^{\mu} \xi^{\nu} \mathrm{d} x^{\alpha} \wedge \mathrm{d} x^{\beta}=\lim _{\mathrm{R} \rightarrow \infty} \frac{1}{8 \pi} \int_{\mathcal{S}_{\mathrm{R}}} \frac{K \partial_{r} K}{\sin \theta} \mathrm{d} \phi \wedge \mathrm{d} \theta=0 .
$$

\footnotetext{
1 The 2-forms in (3.2) are equal on $\mathcal{S}_{\mathrm{R}}$ irrespective of whether Einstein's equations are satisfied or not
} 
We conclude, therefore, that the integral $\lim _{\mathrm{R} \rightarrow \infty} \int_{\mathcal{B}_{\mathbb{R}}} \mathrm{d} m\left(8 \pi \rho^{2}\right)^{-1} e^{-2 \Psi} K \mathcal{L} K$, which was omitted deliberately in (3.1) as it would be zero for $\mathcal{C}^{2}$ solutions, does not vanish. For regularized profiles $K^{\epsilon}$ this integral tends in the limit $\epsilon \rightarrow 0$ to minus the total mass $\int_{\mathbb{R}^{3} \backslash \mathcal{I}} \mathcal{D} \mathrm{d} m$ of the regularity region $\mathbb{R}^{3} \backslash \mathcal{I}$. Putting this in other words, asymptotically flat van Stockum spacetimes contain additional sources of negative active mass located in $\mathcal{I}$. The sources are distributions as they have both finite mass and a spatial support of measure zero.

To be more explicit, the proper energy density $\widetilde{\mathcal{D}}=T_{\mu \nu} u^{\mu} u^{\nu}$, the trace of spatial stresses $\widetilde{\mathcal{S}}=T_{\mu \nu}\left(u^{\mu} u^{\nu}+g^{\mu \nu}\right)$, Tolman's active mass density on a hypersurface of constant time $\widetilde{D}_{T}=(8 \pi)^{-1} R_{\mu}^{t} \xi^{\mu}$, and the curvature scalar $\widetilde{R}=R^{\mu}{ }_{\mu}$ of a spacetime with metric (2.2) read

$$
\begin{gathered}
\widetilde{\mathcal{D}}=\frac{3}{4} e^{-2 \Psi} \frac{K_{, \rho}^{2}+K_{, z}^{2}}{8 \pi \rho^{2}}-\frac{1}{8 \pi} e^{-2 \Psi}\left(\Psi_{, \rho \rho}+\Psi_{, z z}\right), \quad \widetilde{\mathcal{D}}_{T}=e^{-2 \Psi} \frac{K_{, \rho}^{2}+K_{, z}^{2}}{8 \pi \rho^{2}}+\frac{1}{8 \pi} e^{-2 \Psi} \frac{K}{\rho^{2}} \mathcal{L} K, \\
\widetilde{\mathcal{S}}=\frac{1}{4} e^{-2 \Psi} \frac{K_{, \rho}^{2}+K_{, z}^{2}}{8 \pi \rho^{2}}+\frac{1}{8 \pi} e^{-2 \Psi}\left(\Psi_{, \rho \rho}+\Psi_{, z z}\right), \quad \widetilde{\mathcal{R}}=8 \pi(\widetilde{\mathcal{D}}-\widetilde{\mathcal{S}}) .
\end{gathered}
$$

For any smooth $\Psi$ and $K$ satisfying only (2.3), the above definitions reduce to

$$
\begin{gathered}
\widetilde{\mathcal{D}}=\mathcal{D}+e^{-2 \Psi} \frac{K_{, \rho}}{16 \pi \rho} \mathcal{L} K, \quad \widetilde{\mathcal{D}}_{T}=\mathcal{D}+e^{-2 \Psi} \frac{K}{8 \pi \rho^{2}} \mathcal{L} K, \\
\widetilde{\mathcal{S}}=-e^{-2 \Psi} \frac{K, \rho}{16 \pi \rho} \mathcal{L} K, \quad \widetilde{R}=8 \pi \mathcal{D}+e^{-2 \Psi} \frac{K_{, \rho}}{\rho} \mathcal{L} K .
\end{gathered}
$$

In particular, for regularized profiles $K^{\epsilon}$ we obtain, outside $\mathcal{I}_{\delta}$ and in the limit $\epsilon \rightarrow 0, \widetilde{\mathcal{D}}=\widetilde{\mathcal{D}}_{T}=D=(8 \pi)^{-1} \widetilde{R}$ and $\widetilde{\mathcal{S}}=0$, like for dust. However, in the same limit $\int_{\mathcal{I}_{\delta}} \widetilde{S} \mathrm{~d} m \neq 0$ for arbitrarily small $\delta$. Note, that $\mathcal{I}$ is the scalar curvature singularity as $\widetilde{R}$ is a distribution on $\mathcal{I}$. Indeed, if $K_{\epsilon}$ is a regularized profile of an asymptotically flat solution then $\int_{\mathbb{R}^{3}} \widetilde{R} \neq \int_{\mathbb{R}^{3} \backslash \mathcal{I}} \widetilde{R}=8 \pi \int_{\mathbb{R}^{3}} \mathcal{D}$ in the limit $\epsilon \rightarrow 0$. Thus $\widetilde{R}=8 \pi \mathcal{D}+\gamma_{\mathcal{I}}$ where $D$ is smooth and integrable, and $\gamma_{\mathcal{I}}$ is a distribution localized on $\mathcal{I}$. The singularity is not isolated from regularity regions. ${ }^{2}$

Here we give an example illustrating the statements. The Bonnor solution [3] with an embedded surface layer of negative mass, and which we shall denote by $K_{B}^{0}$, can be regularized by defining $K_{B}^{\epsilon}(\rho, z)=\sqrt{8 a^{3} \mu}$. $\rho^{2} \cdot\left(\left(a+\sqrt{z^{2}+\epsilon^{2}}\right)^{2}+\rho^{2}\right)^{-3 / 2}, a>0, \mu>0$. Although $K_{B}^{\epsilon}$ is globally $\mathcal{C}^{\infty}$, its limit $K_{B}^{0}$ is not even differentiable in $\mathcal{I}$, which is the plane $z=0$. On integrating over $\mathbb{R}^{3}$ and taking the limit $\epsilon \rightarrow 0$ we obtain $\int_{\mathbb{R}^{3}} D \mathrm{~d} m=\int_{\mathbb{R}^{3} \backslash \mathcal{I}} D \mathrm{~d} m=\mu, \int_{\mathbb{R}^{3}} \widetilde{D} \mathrm{~d} m=3 \mu / 4, \int_{\mathcal{I}} \widetilde{S} \mathrm{~d} m=\mu / 4, \int_{\mathbb{R}^{3}} \widetilde{R} \mathrm{~d} m=4 \pi \mu$ and $\int_{\mathbb{R}^{3}} \widetilde{D}_{T} \mathrm{~d} m \equiv 0$. The latter holds identically as $8 \pi \sqrt{-g} \widetilde{D}_{T} \mathrm{~d} \rho \wedge \mathrm{d} \phi \wedge \mathrm{d} z=\mathrm{d}\left(\rho^{-1} K K_{, z} \mathrm{~d} \rho \wedge \mathrm{d} \phi+\rho^{-1} K K_{, \rho} \mathrm{d} \phi \wedge \mathrm{d} z\right)$ for $\mathcal{C}^{2}$ functions, hence $\int_{\mathbb{R}^{3}} \widetilde{D}_{T} \mathrm{~d} m \equiv M=0$ for regularized asymptotically flat profiles. Since for $K_{B}^{0} 8 \pi D=\widetilde{R}$ only outside $\mathcal{I}$, and since $\int_{\mathbb{R}^{3} \backslash \mathcal{I}} D \mathrm{~d} m=\int_{\mathbb{R}^{3}} D \mathrm{~d} m=\mu \neq \mu / 2=(8 \pi)^{-1} \int_{\mathbb{R}^{3}} \widetilde{R} \mathrm{~d} m$, the curvature scalar is a distribution, and $\widetilde{R}$ is smooth and bounded only outside $\mathcal{I}$.

These properties and those discussed in the introduction show that global and asymptotically flat van Stockum spacetimes are not viable physically. The same, for example, concerns also asymptotically flat stationary and axisymmetric spacetimes of which internal metrics are matched onto the external line element (2.2), as then $M=0$. However, a possibility that van Stockum spacetime can be part of a regular spacetime cannot be excluded.

\section{THREE CLASSES OF SOLUTIONS}

\section{A. Solutions with a layer of negative mass}

Solutions to equation (2.4) can be sought via integral transforms, for example,

$$
K(\rho, z)=\rho \int_{0}^{\infty} \lambda \hat{K}_{\mathrm{J}}(\lambda) e^{-\lambda|z|} \mathrm{J}_{1}(\lambda \rho) \mathrm{d} \lambda, \quad \text { or } \quad \rho \int_{0}^{\infty} \lambda \hat{K}_{\mathrm{K}}(\lambda) \cos (\lambda z) \mathrm{K}_{1}(\lambda \rho) \mathrm{d} \lambda
$$

generate $z$-symmetric solutions; $\mathrm{J}_{1}$ and $\mathrm{K}_{1}$ are Bessel functions. To give an example, the solution $\rho^{2} r^{-3}$ discussed in [2] has $\hat{K}_{\mathrm{J}}(\lambda)=1$ and $\hat{K}_{\mathrm{K}}(\lambda)=2 / \pi$, while the Bonnor solution $K_{B}^{0}$ has $\hat{K}_{\mathrm{J}}(\lambda) \propto e^{-a \lambda}$. The latter belongs to a class

\footnotetext{
${ }^{2}$ In this sense we cannot agree with the statement of paper [3] that a solution found therein had no curvature singularity.
} 
of solutions defined by specifying $\hat{K}_{\mathrm{J}}(\lambda)=\frac{l^{2 n+2} \lambda^{2 n}}{(2 n+1) !} e^{-a \lambda}, n \in \mathbb{N}$, which yields

$$
K_{n}(\rho, z)=\frac{(n+1) l^{2 n+2} \rho^{2}}{(a+|z|)^{2 n+3}} \cdot{ }_{2} F_{1}\left(\frac{3}{2}+n, 2+n ; 2,-\frac{\rho^{2}}{(a+|z|)^{2}}\right) .
$$

Apart from the plane $z=0$ the solutions are smooth everywhere. One can show that $\left|K_{n}\right|<\rho(l /(a+|z|))^{2 n+2}$, thus, at least for $l<a$, hypersurfaces of constant $t$ are globally space-like as then $\left|K_{n}\right|<\rho$. Since $K_{n} \partial_{r} K_{n}(r, \theta) \sim$ $r^{-(4 n+3)} \sin ^{4} \theta$ times a bounded geometrical factor, the spacetimes are asymptotically flat with $M=0$. Function $\mathcal{D} e^{2 \Psi}$ is finite for $z \neq 0$ and for $r$ sufficiently large behaves as $r^{-(4 n+6)}$. The plane $z=0$ is thus a curvature singularity with finite and negative active mass. Only the Bonnor solution $K_{B}^{0}(n=0)$ has nonzero angular momentum.

\section{B. External and Internal multipolar solutions and a multipole expansion}

Let $K(\rho, z)=W(r) Y(\cos \theta)$, where $\rho=r \sin \theta, z=r \cos \theta$. There exist three families of solutions to equation (2.4) satisfying $r^{2} W^{\prime \prime}(r)=\lambda W(r)$ and $\left(1-x^{2}\right) Y^{\prime \prime}(x)+\lambda Y(x)=0,(x=\cos \theta)$; with i) $\lambda=\alpha(\alpha+1)$, $\alpha \geq 0$; ii) $-\cos ^{2}(\alpha) / 4,0 \leq \alpha<\pi / 2$ and iii) $-\cosh ^{2}(\alpha) / 4, \alpha>0$. The $\left.i\right)$ class contains $x$-analytic external $\left(W=r^{-n}\right)$ and internal $\left(W=r^{n+1}\right)$ solutions. In this way we obtain external $K_{E}^{(n)}$ and internal $K_{I}^{(n)}$ multipolar solutions

\begin{tabular}{|c|c|c|}
\hline$n$ & $0,2,4,6, \ldots$ & $1,3,5,7, \ldots$ \\
\hline$K_{E}^{(n)}(\rho, z)$ & $\frac{z}{\left(\rho^{2}+z^{2}\right)^{\frac{n+1}{2}}} A_{n}\left(\frac{z^{2}}{\rho^{2}+z^{2}}\right)$ & $\frac{1}{\left(\rho^{2}+z^{2}\right)^{n / 2}} B_{n}\left(\frac{z^{2}}{\rho^{2}+z^{2}}\right)$ \\
\hline$K_{I}^{(n)}(\rho, z)$ & $z\left(\rho^{2}+z^{2}\right)^{n / 2} A_{n}\left(\frac{z^{2}}{\rho^{2}+z^{2}}\right)$ & $\left(\rho^{2}+z^{2}\right)^{(n+1) / 2} B_{n}\left(\frac{z^{2}}{\rho^{2}+z^{2}}\right)$ \\
\hline
\end{tabular}

where $A_{n}(y)={ }_{2} F_{1}\left(\frac{1}{2}+\frac{n}{2},-\frac{n}{2} ; \frac{3}{2}, y\right)$ and $B_{n}(y)={ }_{2} F_{1}\left(-\frac{1}{2}-\frac{n}{2}, \frac{n}{2} ; \frac{1}{2}, \frac{z^{2}}{\rho^{2}+z^{2}}\right)$. Internal solutions $K_{I}^{(n)}$ (of which element is the van Stockum solution [1]) give rise to spacetimes that are not asymptotically flat. With the exception of the monopole $K_{E}^{(0)}, K_{E}^{(n)}$ yield asymptotically flat spacetimes that contain in the center pathological singularities with non-integrable $\mathcal{D}$ (e.g. $e^{2 \Psi} \mathcal{D}>a^{4} /\left(2 \pi r^{6}\right)$ for the dipole $\left.K_{E}^{(1)}=a^{2} \sin ^{2}(\theta) r^{-1}\right)$. For the solutions $M=0$, thus contributions to $M$ from the singularities are formally $-\infty$. Another non-physical property of $K_{E}^{(n)}$ is that $|K(\rho, z)|>\rho$ in the vicinity of the center. In the region the axial symmetry Killing vector $\boldsymbol{\eta}$ is time-like, as so, the region contains closed time-like curves (e.g. $K_{E}^{(1)}$ is such inside the region bounded by $\rho=a \sqrt{\sin \alpha} \sin \alpha, z=a \cos \alpha \sqrt{\sin \alpha}, \alpha \in(0, \pi)$ ). However, external multipoles $K_{E}^{(n>0)}$ appear in multipolar expansions of asymptotically flat solutions. We illustrate this by giving an example below.

It should be clear that $K_{a}=-\int_{-a}^{a} a^{-1} s \mathrm{~d} s f(\rho, z-s), a>0$, where $f(\rho, z)=K_{E}^{(0)}=z r^{-1}$, is a $z$-symmetric solution such that $0 \leq K_{a} \leq a$. The conformal mapping $z+i \rho=a \cosh (u+i v)(u \geq 0,0 \leq v \leq \pi)$ is invertible apart from two points $(\rho, z)=(0, \pm a)$ and transforms $S_{a}=\{(\rho, z): \rho=0, z \in[-a, a]\}$ to the segment $u=0, v \in[0, \pi]$. In this map the solution reads

$$
K_{a}(u, v)=a \sin ^{2}(v)\left(\cosh (u)+\frac{1}{2} \sinh ^{2}(u) \ln \left[\tanh ^{2}\left(\frac{u}{2}\right)\right]\right) .
$$

The resulting spacetime is asymptotically flat as $K_{a} \sim(4 / 3) a e^{-u} \sin ^{2} v$ for large $u$. In the vicinity of $u=0$, $K_{a} \sim u^{2} \ln u$, therefore $\mathcal{I}=\mathcal{S}_{a}$. Multipolar expansion of $K_{a}$ in the base of functions $K_{E}^{(n)}, n=1,3,5, \ldots$, reads

$$
K_{a}(\rho, z)=\frac{2}{3} \frac{\rho^{2}}{r^{3}} a^{2}-\frac{1}{5} \frac{\rho^{2}\left(\rho^{2}-4 z^{2}\right)}{r^{7}} a^{4}+\frac{3}{28} \frac{\rho^{2}\left(\rho^{4}-12 \rho^{2} z^{2}+8 z^{4}\right)}{r^{11}} a^{6}+\ldots,
$$

and $\Psi_{a}(r, \theta) \sim-\left(a^{4} / 72\right) r^{-4}(7+9 \cos 2 \theta) \sin ^{2} \theta$ as $r \rightarrow \infty$. Asymptotically, (2.2) reduces to

$$
\mathrm{d} s^{2} \sim-\mathrm{d} t^{2}-\frac{4}{3} \frac{a^{2}}{r} \sin ^{2} \theta \mathrm{d} t \mathrm{~d} \phi+\mathrm{d} r^{2}+r^{2}\left(\mathrm{~d} \theta^{2}+\sin ^{2} \theta \mathrm{d} \phi^{2}\right) .
$$

Comparison with the asymptotic expansion of the Kerr metric gives total mass $M=0$ and total angular momentum $J=a^{2} / 3$ in agreement with (3.2) and with the analogous expression for the total angular momentum of asymptotically flat van Stockum flow

$$
J=\lim _{r \rightarrow \infty} \frac{1}{16 \pi} \iint\left[\frac{2 K}{r}-\left(1+\frac{K^{2}}{r^{2} \sin ^{2} \theta}\right) \partial_{r} K\right] r^{2} \sin \theta \mathrm{d} \theta \mathrm{d} \phi
$$


[1] W.J. van Stockum, Proc. Roy. Soc. Edin. 57, 135 (1937)

[2] W. B. Bonnor, J. Phys. A: Math. Gen. 10, 10, 1673 (1977)

[3] W. B. Bonnor, Gen. Relativ. Gravit. 37, 12, 2245 (2005)

[4] R. M. Wald, General Relativity, The University of Chicago Press (1984) 